QMW-PH-96-17

\title{
Linear quantum state diffusion for non-Markovian open quantum systems
}

\author{
Walter T. Strunz * \\ Department of Physics, Queen Mary and Westfield College, University of London, Mile End \\ Road, London E1 4NS, United Kingdom
}

(October 7, 1996)

\begin{abstract}
We demonstrate the relevance of complex Gaussian stochastic processes to the stochastic state vector description of non-Markovian open quantum systems. These processes express the general Feynman-Vernon path integral propagator for open quantum systems as the classical ensemble average over stochastic pure state propagators in a natural way. They are the coloured generalization of complex Wiener processes in quantum state diffusion stochastic Schrödinger equations.

03.65.Bz, 05.40.+j, 42.50.Lc
\end{abstract}

Typeset using REVTEX

*e-mail: W.Strunz@qmw.ac.uk 


\section{INTRODUCTION}

The reduced density operator of a quantum subsystem is obtained from the total density operator by tracing over the environmental degrees of freedom. Feynman and Vernon [1] derive the propagator $\mathcal{J}\left(x_{f}, x_{f}^{\prime}, t ; x_{0}, x_{0}^{\prime}, 0\right)$ of the reduced density matrix $\rho\left(x, x^{\prime}, t\right)$ in terms of a double path integral [see also Feynman and Hibbs [2]]

$$
\mathcal{J}\left(x_{f}, x_{f}^{\prime}, t ; x_{0}, x_{0}^{\prime}, 0\right)=\int_{x_{0}, 0}^{x_{f}, t} \mathcal{D}[x] \int_{x_{0}^{\prime}, 0}^{x_{f}^{\prime}, t} \mathcal{D}\left[x^{\prime}\right] \exp \left\{\frac{i}{\hbar}\left(S[x]-S\left[x^{\prime}\right]\right)\right\} \mathcal{F}\left[x, x^{\prime}\right]
$$

where $S[x]$ is the classical action functional of the subsystem alone. The influence functional $\mathcal{F}\left[x, x^{\prime}\right]$ combines the effects of the environmental initial state, its Hamiltonian and the interaction Hamiltonian between subsystem and environment, on the subsystem.

It is also shown in [1,2] that the general influence functional which is at most quadratic in the coordinates must be of the form

$$
\mathcal{F}\left[x, x^{\prime}\right]=\exp \left\{-\int_{0}^{t} d \tau \int_{0}^{\tau} d \sigma\left[x_{\tau}-x_{\tau}^{\prime}\right]\left[\alpha(\tau, \sigma) x_{\sigma}-\alpha^{*}(\tau, \sigma) x_{\sigma}^{\prime}\right]\right\}
$$

with a positive, Hermitian kernel

$$
\alpha(\tau, \sigma)=\alpha^{*}(\sigma, \tau)
$$

\section{A. Real kernels}

Feynman and Vernon [1,2] emphasize that if the kernel is not only Hermitian but real, the influence functional can be obtained from a real Gaussian stochastic process $F(\tau)$ (a fluctuating force), with statistical properties

$$
\begin{aligned}
\langle F(\tau)\rangle & =0 \\
\langle F(\tau) F(\sigma)\rangle & =\alpha(\tau, \sigma)=\alpha^{*}(\tau, \sigma) .
\end{aligned}
$$

Here, and throughout the paper, $\langle\ldots\rangle$ denotes the classical ensemble average over the stochastic processes. Using the general formula 


$$
\left\langle\exp \int_{0}^{t} d \tau[f(\tau) F(\tau)]\right\rangle=\exp \frac{1}{2} \int_{0}^{t} d \tau \int_{0}^{t} d \sigma[f(\tau) \alpha(\tau, \sigma) f(\sigma)]
$$

for arbitrary functions $f(\tau)$, the propagator $\mathcal{J}$ for the density operator can be stochastically decoupled into stochastic pure-state propagators $G_{F}$,

$$
\mathcal{J}\left(x_{f}, x_{f}^{\prime}, t ; x_{0}, x_{0}^{\prime}, 0\right)=\left\langle G_{F}\left(x_{f}, t ; x_{0}, 0\right) G_{F}^{*}\left(x_{f}^{\prime}, t ; x_{0}^{\prime}, 0\right)\right\rangle
$$

with

$$
G_{F}\left(x_{f}, t ; x_{0}, 0\right)=\int_{x_{0}, 0}^{x_{f}, t} \mathcal{D}[x] \exp \left\{\frac{i}{\hbar} S[x]+i \int_{0}^{t} d \tau x_{\tau} F(\tau)\right\}
$$

The total action functional in the exponent of the path integral propagator (7) now represents the additional influence of the stochastic force $\hbar F(\tau)$ on the subsystem. Thus, real kernels are equivalent to ordinary unitary, but stochastic, quantum dynamics.

\section{B. Complex kernels}

Genuine environments, however, not only induce fluctuations in the subsystem but also dissipation. These are represented by complex kernels $\alpha(\tau, \sigma)$ and can therefore not be

simulated by a stochastic potential. As an example, Feynman and Vernon [1,2] derive the influence functional (2) analytically for the case of a linear coupling to a heat bath of harmonic oscillators with temperature $T$. Caldeira and Leggett further elaborate this approach in [3] [see also Grabert [4] and Weiss [5]], resulting in the complex quantum Brownian motion kernel

$$
\alpha(\tau, \sigma)=\frac{\gamma m}{\pi \hbar} \int_{0}^{\Omega} d \omega \omega\left\{\operatorname{coth}\left(\frac{\hbar \omega}{2 k T}\right) \cos (\omega(\tau-\sigma))-i \sin (\omega(\tau-\sigma))\right\},
$$

where $\Omega$ is a bath cut-off frequency, $m$ the mass of the particle and $\gamma$ the damping rate.

Remarkably, it has been shown only recently by Diósi [6] that even in the general case of a complex kernel like (8), the Feynman-Vernon propagator (1) allows a stochastic decoupling similar to (6). His result is based on the tricky construction of a real Gaussian process, whose correlation function has been given implicitly in terms of $\alpha$. From the point of view 
of applications, however, the use of these processes appears rather difficult. The deeper reason behind this construction comes from relativistic measurement theory [7].

It is the aim of this paper to present an alternative and much simpler stochastic decoupling of the general Feynman-Vernon influence functional, based on complex Gaussian stochastic processes. In their white noise version, these processes have been introduced from symmetry considerations in the quantum state diffusion (QSD) stochastic Schrödinger equation [8 12], describing Markovian open quantum systems. They also appear in measurement

theories, particularly in cases where the apparatus is represented by a Bosonic reservoir [see 13. for more references].

Markovian stochastic state vector methods have proven indispensable for many applications, particularly in quantum optics [14. Our result represents a first step towards an applicable non-Markovian stochastic state vector theory, required for instance in solid state theory 4.5 . 5 .

In a recent related work Kleinert and Shabanov [15], derive operator quantum Langevin equations in the Heisenberg picture corresponding to the propagator (11). In this paper, however, we stick to path integrals and state vectors in the Schrödinger picture. We review basic properties of complex Gaussian processes in Sect. 2. In Sect. 3 we show how they enable the stochastic decoupling of the general Feynman-Vernon propagator, resulting in linear non-Markovian quantum state diffusion. We conclude with a short discussion and further comments in the final Sect. 4.

\section{COMPLEX GAUSSIAN STOCHASTIC PROCESSES}

Here we review complex Gaussian processes $Z(\tau)$ with stochastic properties

$$
\begin{aligned}
\langle Z(\tau)\rangle & =0 \\
\langle Z(\tau) Z(\sigma)\rangle & =0 \text { and } \\
\left\langle Z(\tau) Z^{*}(\sigma)\right\rangle & =\gamma(\tau, \sigma) .
\end{aligned}
$$


Such processes $Z(\tau)$ can only be constructed if the complex correlation $\gamma$ is Hermitian and positive, which is automatically fulfilled by the quantum environments we are interested in. The processes $Z(\tau)$ with properties (9) are the natural coloured generalization of complex Wiener processes $\xi(\tau)$ with corresponding Itô increments $d \xi$ with properties

$$
(d \xi)^{2}=0 \text { and }|d \xi|^{2}=d t
$$

as they arise from symmetry considerations in the quantum state diffusion theory of Markovian open quantum systems [8 11]. Complex processes with properties (9) are also common in quantum measurement theories [13].

Writing $Z(\tau)=X(\tau)+i Y(\tau)$ we find that conditions (9) are fulfilled for

$$
\begin{gathered}
\langle X(\tau) X(\sigma)\rangle=\langle Y(\tau) Y(\sigma)\rangle=\frac{1}{2} \operatorname{Re}\{\gamma(\tau, \sigma)\} \text { and } \\
\langle X(\tau) Y(\sigma)\rangle=-\langle Y(\tau) X(\sigma)\rangle=-\frac{1}{2} \operatorname{Im}\{\gamma(\tau, \sigma)\} .
\end{gathered}
$$

We see that the crucial advantage of complex processes is to allow a non-vanishing correlation between their real and imaginary part, regarding the real processes $X(\tau)$ and $Y(\tau)$ as one joint real Gaussian process $(X(\tau), Y(\tau))$. This construction leads to the imaginary part of the correlation function $\gamma(\tau, \sigma)$ in $(9)$.

The relevant formula for complex processes $Z(\tau)$ that replaces formula (5) for real processes is

$$
\left\langle\exp \int_{0}^{t} d \tau\left[f(\tau) Z(\tau)+g(\tau) Z^{*}(\tau)\right]\right\rangle=\exp \int_{0}^{t} d \tau \int_{0}^{t} d \sigma[f(\tau) \gamma(\tau, \sigma) g(\sigma)]
$$

valid for arbitrary functions $f(\tau)$ and $g(\tau)$. Notice that this implies

$$
\left\langle\exp \int_{0}^{t} d \tau[f(\tau) Z(\tau)]\right\rangle=1
$$

in contrast to equation (5) for real processes.

If one wants to generate such processes $Z(\tau)$ numerically, one can use the construction

$$
Z(t)=\int d \tau \gamma_{\frac{1}{2}}(t, \tau) \xi(\tau)
$$


where $\xi(\tau)$ is an easily generated white complex process and

$$
\int d \tau \gamma_{\frac{1}{2}}(t, \tau) \gamma_{\frac{1}{2}}(\tau, s)=\gamma(t, s)
$$

\section{STOCHASTIC DECOUPLING OF THE FEYNMAN-VERNON INFLUENCE FUNCTIONAL}

We show how the processes $Z(\tau)$ naturally lead to a stochastic decoupling of the Feynman-Vernon influence functional. We choose processes with the complex conjugate of the Feynman-Vernon kernel from (2) as correlation function,

$$
\gamma(\tau, \sigma)=\alpha^{*}(\tau, \sigma)
$$

According to formula (12) we find

$$
\begin{aligned}
\left\langle\exp \int_{0}^{t} d \tau\left[x_{\tau} Z(\tau)+x_{\tau}^{\prime} Z^{*}(\tau)\right]\right\rangle & =\exp \int_{0}^{t} d \tau \int_{0}^{t} d \sigma\left[x_{\tau} \alpha^{*}(\tau, \sigma) x_{\sigma}^{\prime}\right] \\
& =\exp \int_{0}^{t} d \tau \int_{0}^{\tau} d \sigma\left[x_{\tau} \alpha^{*}(\tau, \sigma) x_{\sigma}^{\prime}+x_{\tau}^{\prime} \alpha(\tau, \sigma) x_{\sigma}\right]
\end{aligned}
$$

where we used (3) to get the second line. This last expression is just the part of the influence functional (2) that couples $x$ and $x^{\prime}$. Notice, however, that they are decoupled on the lefthand side of equation (17). We conclude that we can express the propagator of the density matrix in the decoupled form

$$
\mathcal{J}\left(x_{f}, x_{f}^{\prime}, t ; x_{0}, x_{0}^{\prime}, 0\right)=\left\langle G_{Z}\left(x_{f}, t ; x_{0}, 0\right) G_{Z}^{*}\left(x_{f}^{\prime}, t ; x_{0}^{\prime}, 0\right)\right\rangle
$$

with the stochastic path integral propagator for state vectors,

$$
G_{Z}\left(x_{f}, t ; x_{0}, 0\right)=\int_{x_{0}, 0}^{x_{f}, t} \mathcal{D}[x] \exp \left\{\frac{i}{\hbar} S[x]+\int_{0}^{t} d \tau x_{\tau} Z(\tau)-\int_{0}^{t} d \tau \int_{0}^{\tau} d \sigma\left[x_{\tau} \alpha(\tau, \sigma) x_{\sigma}\right]\right\}
$$

Thus, the Feynman-Vernon path integral propagator (1) for the density operator is equivalent

to the ensemble of pure state propagators (19). This is the main result of this paper. The non-local action functional in (19) reflects the non-Markovian nature of the problem. 
Result (18) with (19) allows to describe the non-Markovian dynamics of the subsystem in terms of an ensemble of stochastic state vectors

$$
\left|\psi_{Z}(t)\right\rangle=G_{Z}(t ; 0)\left|\psi_{0}\right\rangle
$$

If we assume an initial pure state

$$
\rho_{0}=P_{\psi_{0}}
$$

where we use the notation $P_{\psi}=|\psi\rangle\langle\psi|$ for pure state projectors, we recover the density operator at time $t$ by taking the ensemble average according to (18),

$$
\rho(t)=\left\langle P_{\psi_{Z}(t)}\right\rangle
$$

In Markovian subsystem dynamics [8 11], the time evolution of the stochastic state vectors is given by a stochastic Schrödinger equation. In fact, in the white noise case

$$
\alpha(t, s)=\kappa \delta(t-s)
$$

the path integral propagator (19) becomes local in time,

$$
G_{\xi}\left(x_{f}, t ; x_{0}, 0\right)=\int_{x_{0}, 0}^{x_{f}, t} \mathcal{D}[x] \exp \left\{\frac{i}{\hbar} S[x]+\sqrt{\kappa} \int_{0}^{t} d \xi(\tau) x_{\tau}-\frac{\kappa}{2} \int_{0}^{t} d \tau x_{\tau}^{2}\right\},
$$

with a delta-correlated normalized complex process $\xi(t)=\kappa^{-\frac{1}{2}} Z(t)$. This is the propagator of the linear quantum state diffusion stochastic Schrödinger equation

$$
|d \psi\rangle=\left(-\frac{i}{\hbar} \hat{H}-\frac{\kappa}{2} \hat{x}^{2}\right)|\psi\rangle d t+\sqrt{\kappa} \hat{x}|\psi\rangle d \xi
$$

with complex Itô increments (10). The path integral theory of general linear quantum state diffusion equations was developed in [12], where a rigorous definition of stochastic path integrals like (24) is given.

As the non-Markovian generalization of (24), the propagator (19) represents linear nonMarkovian quantum state diffusion. 


\section{CONCLUSIONS}

We use complex Gaussian stochastic processes to find a non-Markovian quantum state diffusion theory which is equivalent to the Feynman-Vernon density matrix formulation. Our

result offers a helpful tool, since state vectors are simpler than density operators. Such a reduction in complexity is essential to tackle many realistic problems and is most significant numerically, as is well recognized in the Markovian case, for instance in quantum optics [14]. To be truly helpful, one must overcome the difficulties arising from the fact that the stochastic state vectors $\left|\psi_{Z}\right\rangle$ of $(20)$ are not normalized. Also an effective algorithm to propagate state vectors with the non-local path integral (19) has to be established.

From a quantum foundational point of view the question arises in what sense the stochastic state vectors $\left|\psi_{Z}\right\rangle$ can be related to the non-Markovian dynamics of individual open quantum systems.

In the well established Markovian case, the use of white complex noise arose from symmetry considerations in the space of the environment operators of the corresponding master equation [11]. For a single, Hermitian environment operator like $\hat{x}$ in (25), however, this symmetry argument does not apply. In this paper we have shown that even in this case, complex Gaussian processes appear indispensable as soon as non-Markovian features are taken into account. These independent arguments underline their relevance to a stochastic description of general open quantum systems.

\section{ACKNOWLEDGMENT}

I would like to thank Ian C. Percival for helpful discussions and advice. I am also grateful to Lajos Diósi and Todd A. Brun for detailed comments on the manuscript. This work was made possible by a Feodor Lynen fellowship of the Alexander von Humboldt foundation. 


\section{REFERENCES}

[1] R. P. Feynman and F. L. Vernon, Ann. Phys. 24, 118 (1963).

[2] R. P. Feynman and A. R. Hibbs, Quantum mechanics and path integrals (McGraw-Hill, New York, 1965).

[3] A. O. Caldeira and A. J. Leggett, Physica A 121, 587 (1983).

[4] H. Grabert et al., Phys.Rep. 168, 115 (1988).

[5] U. Weiss, Quantum dissipative systems (World Scientific, Singapore, 1993).

[6] L. Diósi, in Stochastic evolution of quantum states in open quantum systems and in measurement processes, eds. L. Diósi and B. Lukács, (World Scientific, Singapore, 1994).

[7] L. Diósi, Phys. Rev. A 42, 5086 (1990).

[8] N. Gisin and I. C. Percival, Phys. Lett. A 167, 315 (1992).

[9] N. Gisin and I. C. Percival, J.Phys.A: Math. Gen. 25, 5677 (1992).

[10] N. Gisin and I. C. Percival, J.Phys.A: Math. Gen. 26, 2233 (1993).

[11] N. Gisin and I. C. Percival, J.Phys.A: Math. Gen. 26, 2245 (1993).

[12] W. T. Strunz, Phys. Rev. A 54, 2664 (1996).

[13] V. P. Belavkin et. al. (eds.), Quantum communications and measurement, (Plenum Press, New York, 1995).

[14] H. Carmichael, An open system approach to quantum optics (Springer, Berlin, 1994).

[15] H. Kleinert and S.V. Shabanov, Phys. Lett. A 200, 224 (1995). 